\title{
Antitumor effect of a polysaccharide isolated from Phellinus pullus as an immunostimulant
}

\author{
WEIHUA YANG ${ }^{1}$, HENGLAN ZHANG $^{2,3}$, MINGYU JI $^{1}$, FENGYAN PEI $^{1}$ and YUNSHAN WANG ${ }^{1}$ \\ ${ }^{1}$ Diagnosis Center of Medical Laboratory, Jinan Central Hospital Affiliated to Shandong University, \\ Jinan, Shandong 250013; ${ }^{2}$ Department of Oncology, The Third People's Hospital of Jinan, Jinan, Shandong 250132; \\ ${ }^{3}$ The Institute of Basic Medicine, Shandong Academic of Medicine Science, Jinan, Shandong 250062, P.R. China
}

Received August 14, 2015; Accepted January 13, 2016

DOI: $10.3892 /$ br.2016.587

\begin{abstract}
The antitumor function of fungal polysaccharides is a popular area of interest in the research field due to their high efficiency and low side effects. The main mechanism of fungal polysaccharides is immune enhancement. The polysaccharose (APS-3) was extracted from the fruit body of Phellinus pullus. The proliferation inhibition to mouse sarcoma 180 (S180) tumor cells was studied by the MTT method. Mice models of transplanted S180 tumor were established and treated with APS-3 to verify the antitumor activity in vivo. Natural killer (NK) and lymphokine-activated killer (LAK) cytotoxicities of the mice were evaluated by the lactate dehydrogenase method. APS-3 can significantly inhibit the proliferation of the S180 cells. Cells could be completely inhibited by $1.6 \mathrm{mg} / \mathrm{ml}$ APS-3 after $24 \mathrm{~h}$ treatment. After 18 days of treatment, the antitumor rate of the high-dose group was $85.47 \%$. Histopathology detection showed that for the APS-3-treated mice, the tumor cells dissolved, and exhibited a large range of structureless necrotic areas. NK and LAK cytotoxicities of the APS-3 treated mice increased by 61.85 and $56.16 \%$, respectively, compared with the normal control mice. APS-3 can be used as an antitumor agent by way of immune enhancement.
\end{abstract}

\section{Introduction}

In conventional tumor therapies, chemotherapy, radiotherapy and surgical treatment all have limitations and side effects that affect normal cells and endanger to the immunity system $(1,2)$. Patients even succumb of serious side effects, such as myelotoxicity and myocardial injury. To search for novel effective antitumor agents with less toxic effects, natural products have been focused on previously $(3,4)$. In the 1960s, the antitumor

Correspondence to: Professor Yunshan Wang, Diagnosis Center of Medical Laboratory, Jinan Central Hospital Affiliated to Shandong University, 105 Jiefang Road, Jinan, Shandong 250013, P.R. China E-mail: sdjnwys@163.com

Key words: Phellinus pullus, polysaccharides, antitumor, S180, immune enhancement function of fungal polysaccharides was discovered and became a popular area of the research field (5). Since then, it has been widely studied owing to the immunostimulating and low toxicity side effects in the host. Fungal polysaccharides, such as lentinan and Ganoderma lucidum polysaccharide have previously been used in clinical cancer therapies (6).

Natural killer (NK) cells are innate lymphocytes that are capable of eliminating tumor cells and are therefore used for cancer therapy (7). Lymphokine-activated killer (LAK) cells are activated NK cells that are traditionally prepared from isolated T cells cultured with interleukin-2 (IL-2) and are capable of recognizing cancer cells in a non-major histocompatibility complex-restricted manner (8). LAK cells have been utilized in vivo in animals and in humans to treat cancer and can kill NK-non-sensitive tumor cells (9).

Phellinus pullus is a wood rot fungi widely distributed in China that has not been used as a traditional herbal medicine. To the best of our knowledge, there are no pharmacological studies on Phellinus pullus. The present study isolated a crude polysaccharide (APS-3) from Phellinus pullus. Its antitumor activity was evaluated in vitro and in vivo. In addition, the immunomodulatory effects of APS-3 were also assessed to analyze the underlying mechanisms of its antitumor activity.

\section{Materials and methods}

Ethical statement. The present study was carried out in strict accordance with the recommendations of the Ethical Review Committee of Affiliate Jinan Central Hospital Affiliated to Shandong University (Shandong, China).

Preparation of polysaccharoses. The polysaccharose APS-3 was obtained from the fruiting bodies of wildly grown Phellinus pullus in the Shandong province of China. The dry fruit body was cleaned, crushed and extracted with boiling $\mathrm{NaOH}(1.5 \%)$ for $30 \mathrm{~min}$ three times. The extract was combined and precipitated with $80 \%$ ethanol. The precipitates were collected and dissolved with distilled water. The Savage method (10) and activated carbon were used to remove protein and pigment from the extract, and subsequently the polysaccharide APS-3 was obtained. The APS-3 content was measured by the phenol-sulfate acid method and was prepared as a $2-\mathrm{mg} / \mathrm{ml}$ stock solution. The stock solution was sterilized 
using a $0.45-\mu \mathrm{m}$ filter (Pall Corporation, Port Washington, NY, USA), and further diluted with cell culture medium to the defined concentrations as indicated.

Cell culture and growth inhibition test. Mouse sarcoma 180 (S180) tumor cells (Cell Bank of Type Culture Collection of Chinese Academy of Sciences, Shanghai, China) were cultured and harvested from ascites of the infected mouse. In vitro culture was performed in RPMI-1640 medium (Gibco Life Technologies, Thermo Fisher Scientific, Inc., Carlsbad, CA, USA) supplemented with $10 \%$ fetal bovine serum (Zhejiang Tianhang Biological Technology Co., Ltd., Huzhou, China), and $300 \mathrm{mg} / 1$ L-glutamine (Gibco Life Technologies, Thermo Fisher Scientific, Inc.), and antibiotics in a humidified $5 \% \mathrm{CO}_{2}$ at $37^{\circ} \mathrm{C}$. A total of $1 \times 10^{5}$ cells $/ \mathrm{ml}$ of exponentially growing cells were cultured in the medium with $0.1,0.2,0.4$, 0.8 and $1.6 \mathrm{mg} / \mathrm{ml} \mathrm{APS}-3$ for $24 \mathrm{~h}$, and subsequently, cell growth inhibition was tested by the MTT method.

\section{APS-3 treatment in vivo}

Animal treatmentand experimental design. A total of 25 female Swiss mice (18-22 g) were provided by the Laboratory Animal Center of Shandong University [animal license number: SCXK (Lu) 20090001]. The animals were allowed free access to a standard diet and sterile water, and were maintained in a sterile and ventilated room under controlled environmental conditions $\left(25 \pm 1^{\circ} \mathrm{C}, 50 \pm 10 \%\right.$ humidity and 12 -h light/12-h dark cycle).

The exponentially growing S180 cells were washed with Hank's balanced salt solution and adjusted to a suspension containing $1 \times 10^{7}$ cells $/ \mathrm{ml}$ in serum-free RPMI-1640 medium. A total of 20 mice were implanted with $200 \mu 1$ of the cell suspension by subcutaneous injection to the fore right subaxillary. Seven days after tumor cell inoculation, the tumor-bearing mice were randomly divided into 4 groups ( 5 mice in each group): Mice received APS-3 intragastrically at the respective doses of $1.5,3$ and $6 \mathrm{~g} / \mathrm{kg} / \mathrm{day}$ (the 3 treated groups, respectively); and the control mice received the same volume of $0.9 \%$ normal saline (NS) intragastrically (the model control, MC). A total of 5 normal mice treated with only NS served as the normal control (NC). All treatments were administered once daily for 18 consecutive days.

Determination of survival time. After 18 days of treatment, the number of surviving mice in each group was recorded and the percentage of life prolongation was calculated as follows: Life prolongation rate $(\%)=$ (survival time of the treated group - survival time of the MC)/survival time of the model control x 100.

Tumor inhibition rate. Tumor size was measured daily and the tumor volume was calculated using the following formula: $\mathrm{V}=0.5 \mathrm{ab} 2$, where $\mathrm{a}$ is the largest and $\mathrm{b}$ is the smallest perpendicular diameter. After 18 days of treatment, all the mice were euthanized, and the tumors were removed and weighed. The tumor inhibition rate was calculated as follows: Inhibition rate $(\%)=($ mean tumor weight in the model control mice - mean tumor weight in the treated mice)/mean tumor weight in the model control mice x 100.

Histopathology. Tumor tissues were fixed in formalin, paraffin-embedded and subsequently separated into sections. The difference in the tumor tissues between the model control mice and the treated group mice was assessed by histopathological analysis following staining with hematoxylin and eosin (H\&E).

\section{Immune-stimulant function of APS-3}

Evaluation of NK cytotoxicity. Spleens of the mice were removed under sterile conditions, disaggregated in D-hanks and filtered through a 200-mesh stainless-steel sieve to obtain a single-cell suspension. Lymphocytes were collected and suspended in RPMI-1640 medium at a concentration of $1 \times 10^{6}$ cells $/ \mathrm{ml}$ following treatment with red blood cell lysis buffer. The activity of NK cells was tested using the cytotoxicity assay on YAC-1 cells (Cell Bank of Type Culture Collection of Chinese Academy of Sciences). A total of $100 \mu \mathrm{l} \mathrm{NK}$ and YAC-1 cells were added to a 96-well plate in triplicate to obtain an effector/target $(\mathrm{E} / \mathrm{T})$ ratio of $20: 1$ and co-incubated for $12 \mathrm{~h}$. The amount of released lactate dehydrogenase (LDH) in culture supernatants was determined using the LDH Cytotoxicity assay kit (Biovision, Inc., Milpitas, CA, USA) according to the manufacturer's protocol. The optical density (OD) was read at $490 \mathrm{~nm}$ with a microplate reader. The percentage of NK cell cytotoxicity was calculated with the formula: Cytotoxicity $(\%)=($ experimental release - spontaneous release)/(maximum release - spontaneous release) x 100 . Spontaneous release was spontaneous LDH release from target cells incubated with medium alone, and maximum release was obtained from target cells lysed with NP-40.

Evaluation of LAK cytotoxicity. Lymphocytes were collected at a concentration of $1 \times 10^{6}$ cells $/ \mathrm{ml}$, as described for the NK cells, and were cultured in the medium with $1,000 \mathrm{U} / \mathrm{ml}$ of IL-2. The cells collected by centrifuge $72 \mathrm{~h}$ later were LAK cells. The concentration of the LAK cells was adjusted to $2 \times 10^{6}$ cells $/ \mathrm{ml}$. Mouse mast cell tumor P815 cells (Cell Bank of Type Culture Collection of Chinese Academy of Sciences) were used as target cells and the concentration was $1 \times 10^{6}$ cells $/ \mathrm{ml}$. LAK cells and P815 cells were co-incubated for $24 \mathrm{~h}$ with an $\mathrm{E} / \mathrm{T}$ ratio of 20:1. A single effector cell and single target cell were used as controls. Each well was measured with the MTT method. The percentage of LAK cell cytotoxicity was calculated with the formula: Cytotoxicity $(\%)=\left[1-\left(\mathrm{OD}_{\text {co-incubated effector cell and target }}\right.\right.$ cell $-\mathrm{OD}_{\text {effector cell }} / \mathrm{OD}_{\text {target cell }}$ x 100.

Statistical analysis. Each experiment was performed at least in triplicate. All the results are expressed as the mean \pm standard deviation. The data were analyzed by the Student's unpaired t-test and $\mathrm{P}<0.05$ was considered to indicate a statistically significant difference.

\section{Results}

Growth inhibition of S180 cell by APS-3 in vitro. The proliferation of the S180 cells was evidently inhibited after 24 -h treatment by APS-3 $(\mathrm{P}<0.05)$ (Fig. 1). The results also indicated that there was a marked dose-dependent inhibition of cell viability.

\section{Antitumor effect in vivo}

Determination of survival time. On day 19 of the treatment, the model control mice were ill or had died. However, the majority of the mice in the treated group survived. All doses of APS-3 significantly prolonged survival time of the mice, showing life prolongation ratios of 36,80 and $85 \%$ at APS-3 


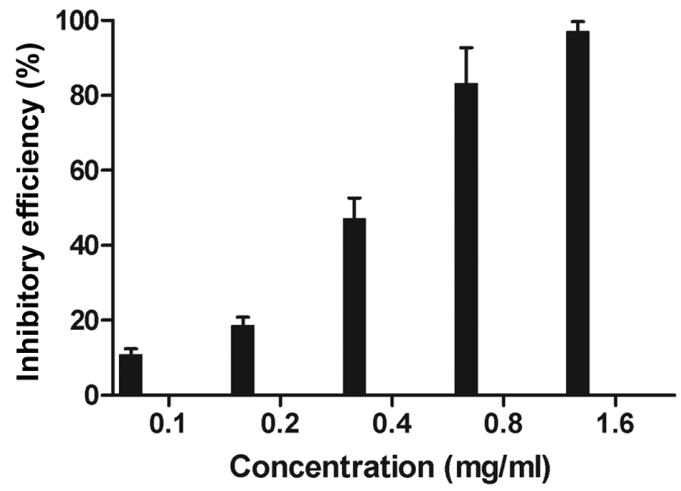

Figure 1. Effect of APS-3 on viability and proliferation of the sarcoma 180 cells. Cells $\left(1 \times 10^{5}\right.$ cells $\left./ \mathrm{ml}\right)$ were incubated with APS-3 at the concentrations of $0.1,0.2,0.4,0.8$ and $1.6 \mathrm{mg} / \mathrm{ml}$ for $24 \mathrm{~h}$. The data are based on the mean of 3 independent experiments, in each of which determinations were in triplicate.

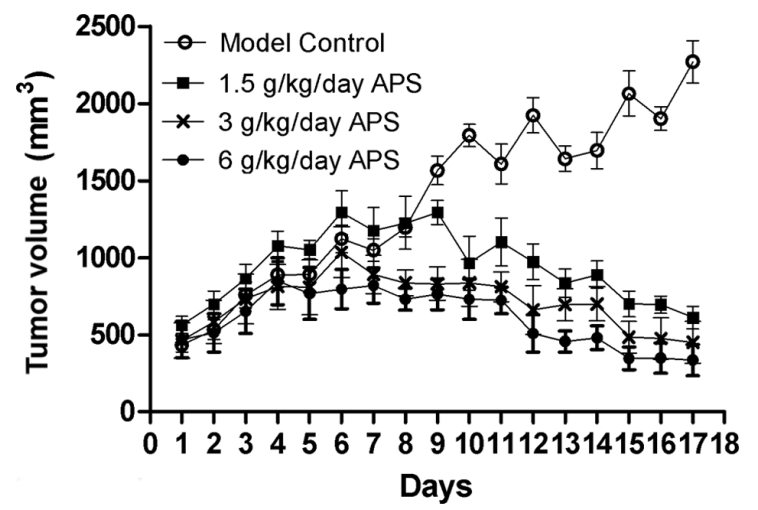

Figure 2. Growth curves in tumor-transplanted mice. Compared with the model control mice, the tumor volume in the treated mice decreased during the first 3 days of treatment, but from day 8 of treatment, the tumor volume decreased significantly.

A

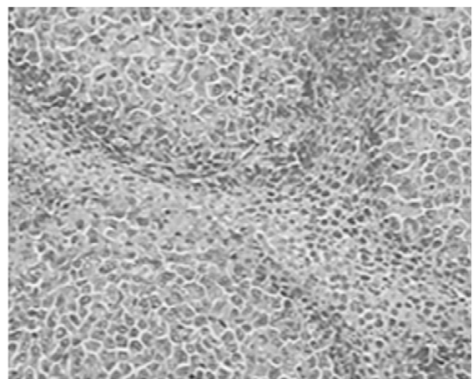

B

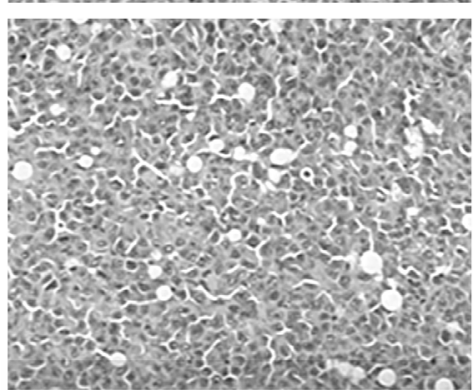

Figure 3. Histopathology of the tumor tissue. Tumor tissues were stained with hematoxylin and eosin and observed under a microscope. In the (A) model control mice, regularly arranged tumor cells were observed, while in the (B) APS-3-treated mice, tumor cells disappeared and inflammatory cell infiltration was observed.

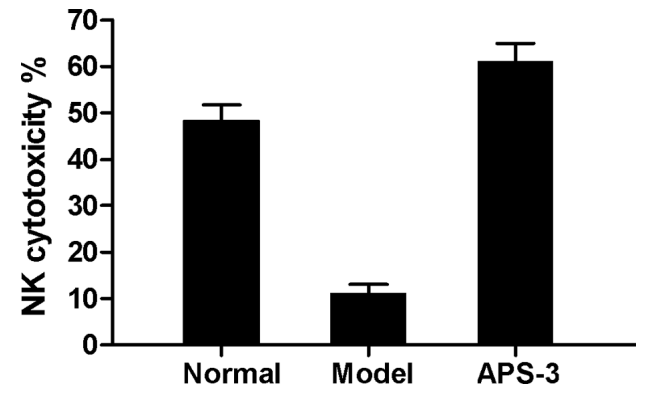

Figure 4. Enhancement of NK cytotoxicity in tumor-bearing mice by APS-3. NK cells were isolated from the spleens of mice with sarcoma 180 tumor and NK cytotoxicity was tested using the lactate dehydrogenase cytotoxicity assay kit. NK cytotoxicity of the APS-3-treated mice was higher compared to the model control mice and normal control mice. NK, natural killer.

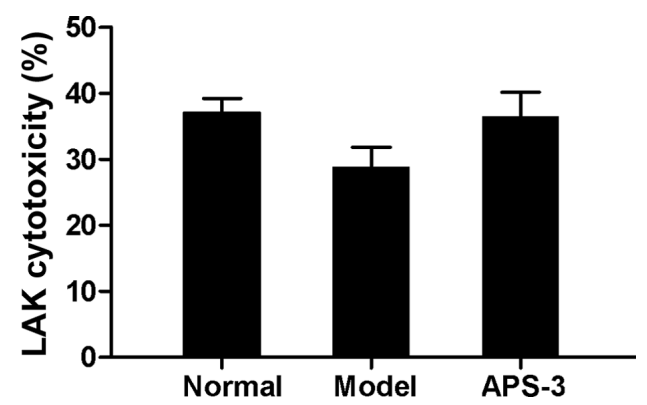

Figure 5. Enhancement of LAK cytotoxicity in tumor-bearing mice by APS-3. LAK cells of the mice with sarcoma 180 tumor were induced and the cytotoxicity of the APS-3 treated mice was higher than that of the model control mice. LAK, lymphokine-activated killer.

doses of $1.5,3$ and $6 \mathrm{~g} / \mathrm{kg} /$ day, respectively. However, as all the mice were sacrificed on day 19 of the treatment, the life prolongation ratios do not signify the final result.

Tumor inhibition rate. Seven days after S180 cell injection, tumors of $\sim 500 \mathrm{~mm}^{3}$ formed in 20 mice. As shown in Fig. 2, APS-3 inhibited tumor growth in a time-dependent manner. The tumor volume decreased after 3 days of APS-3 treatment, but a significant decrease was observed after 9 days of treatment. On day 10 of treatment, the tumor in 1 treated mouse completely disappeared. The tumor volume in the model control mice increased to $1,902 \mathrm{~mm}^{3}$ at day 16 , while the tumors in the APS-3-treated mice were only 995.7, 476.4 and $348.0 \mathrm{~mm}^{3}$, respectively. After APS-3 treatment for 18 days, all mice were sacrificed and the tumors were removed. The tumor weight in the model control mice was $1.79 \mathrm{~g}$, while that in the APS-3-treated mice was only 1.09, 0.35 and $0.26 \mathrm{~g}$ at the dose of $1.5,3$ and $6 \mathrm{~g} / \mathrm{kg} / \mathrm{day}$, and the tumor inhibition ratio was $39.11,80.45$ and $85.47 \%$, respectively.

Histopathology. Fig. 3 shows that in the paraffin sections of the tumor tissues stained with H\&E, regularly arranged tumor cells were observed in the model control mice, and a few necrotic areas and no inflammatory cell infiltrations were observed. However, in the APS-3-treated mice, tumor cells disappeared, and a large range of structureless necrotic areas were observed. Furthermore, inflammatory cell infiltration and granulation tissues were observed in these areas. These findings correlated with the tumor volume and weight in the model control mice and APS-3-treated mice. 
Evaluation of NK cytotoxicity. Following treatment of the tumor-bearing mice with APS-3, NK cell cytotoxicity was tested using the LDH Cytotoxicity assay kit, with YAC-1 cells as the target cells. Splenic lymphocytes of APS-3 treated mice showed more cytotoxicity compared to the splenocytes from the normal mice and model mice $(\mathrm{P}<0.01)$ (Fig. 4). These results indicate that APS-3 increased NK cell cytotoxicity of the tumor-bearing mice.

Evaluation of LAK cytotoxicity. Cytotoxicity of the LAK cells to P815 cells was detected in all the tumor-bearing mice and is shown in Fig. 5. LAK cell cytotoxicity of APS-3-treated mice was not only higher than model control mice, but also higher than NC mice.

\section{Discussion}

Growth of cancer cells can destroy the surrounding environment and release a danger signal, and the immune system of the body can cause the immune response to this danger signal (11). These signals can lead to inflammation, activate the antitumor effector cells and the antigen-presenting cells, triggering the immune response of T cells and B cells $(12,13)$. Immunity of the tumor-burdened body is extremely low, although it is possible to recognize and present the tumor antigen, and produce an immune response (14). Therefore, increasing the immune system of the body to recognize a dangerous signal or activate the effector cells with antitumor activity, such as NK cells and LAK cells, is the main target of tumor immunotherapy (15). Polysaccharide drugs are novel antitumor substances, which exhibit a pharmacology role through numerous channels, and for multi-targets, immune regulation is the main pathway (16-18). For example, the antitumor function of lentinan was by way of generation of activated NK cells $(19,20)$.

The polysaccharide APS-3 was obtained through a decoction extraction using $1.5 \% \mathrm{NaOH}$ and alcohol precipitation of the fruiting bodies of the fungus Phellinus pullus. APS- 3 can inhibit the proliferation of S180 cells in vitro. In vivo studies further confirmed its antitumor effect on S180-transplanted mice tumors, and the highest antitumor rate was $85.47 \%$. Furthermore, the antitumor effect of APS- 3 was dose- and time-dependent. It is possible that if the treatment had been extended, certain mice may have recovered entirely. However, it can significantly improve the activity of NK cells and LAK cells in S180 sarcoma mice, demonstrating that immune enhancement is the main mechanism of the antitumor function of APS-3. In view of the poly-target of fungal polysaccharides, the antitumor function of APS-3 compounds is via numerous links, and a number of targets exhibit its antitumor and immune regulation. Other antitumor mechanisms of APS-3 require further research.

In conclusion, the present study verified the antitumor activity of APS-3, suggesting that APS-3 can be used as a possible candidate for tumor prevention or treatment, and by contrast, APS-3 can be used as an immune-enhancement agent in chemotherapy or surgical treatment.

\section{Acknowledgements}

The authors would like to acknowledge the financial support of the Medical and Health Technology Development Plan of
Shandong province (grant no. 2014WS0002) and the China Postdoctoral Science Foundation (grant no. 2014M551913).

\section{References}

1. Sagar SM, Yance D and Wong RK: Natural health products that inhibit angiogenesis: A potential source for investigational new agents to treat cancer-Part 1. Curr Oncol 13: 14-26, 2006.

2. Wang S, Wu X, Tan M, Gong J, Tan W, Bian B, Chen M and Wang Y: Fighting fire with fire: Poisonous Chinese herbal medicine for cancer therapy. J Ethnopharmacol 140: 33-45, 2012.

3. Nobili S, Lippi D, Witort E, Donnini M, Bausi L, Mini E and Capaccioli S: Natural compounds for cancer treatment and prevention. Pharmacol Res 59: 365-378, 2009.

4. Bao X, Yuan H, Wang C, Liu J and Lan M: Antitumor and immunomodulatory activities of a polysaccharide from Artemisia argyi. Carbohydr Polym 98: 1236-1243, 2013.

5. Kamasuka T, Momoki Y and Sakai S: Antitumor activity of polysaccharide fractions prepared from some strains of Basidiomycetes. Gan 59: 443-445, 1968.

6. Yin X, Ying J, Li L, Zhang H and Wang H. A meta-analysis of lentinan injection combined with chemotherapy in the treatment of nonsmall cell lung cancer. Indian J Cancer 52: E29-E31,2015

7. Lim O, Jung MY, Hwang YK and Shin EC: Present and future of allogeneic natural killer cell therapy. Front Immunol 6: 286, 2015.

8. Helfand SC, Soergel SA, Modiano JF, Hank JA and Sondel PM: Induction of lymphokine-activated killer (LAK) activity in canine lymphocytes with low dose human recombinant interleukin-2 in vitro. Cancer Biother 9: 237-244, 1994.

9. Li Y, Meng FD, Tian X, Sui CG, Liu YP and Jiang YH: Impact of IL-2 and IL-2R SNPs on proliferation and tumor- killing activity of lymphokine-activated killer cells from healthy chinese blood donors. Asian Pac J Cancer Prev 15: 7965-7970, 2014.

10. Zhang WJ: Biochemistry technology of polysaccharide compound. 11-12th edition. Zhejiang University Press, Hangzhou, pp12-21, 1999.

11. Sounni NE and Noel A: Targeting the tumor microenvironment for cancer therapy. Clin Chem 59: 85-93, 2013.

12. Trinchieri G: Cancer Immunity: Lessons From Infectious Diseases. J Infect Dis 212 (Suppl 1): S67-S73, 2015.

13. Guo H, Callaway JB and Ting JP: Inflammasomes: mechanism of action, role in disease, and therapeutics. Nat Med 21: 677-687, 2015.

14. Liu R, Xiong S, Zhang L and Chu Y: Enhancement of antitumor immunity by low-dose total body irradiationis associated with selectively decreasing the proportion and number of T regulatory cells. Cell Mol Immunol 7: 157-162, 2010.

15. West EJ, Scott KJ, Jennings VA and Melcher AA: Immune activation by combination human lymphokine-activated killer and dendritic cell therapy. Br J Cancer 105: 787-795, 2011.

16. Lin CY, Chuang TF, Liao KW, Huang YJ, Pai CC and Chu RM: Combined immunogene therapy of IL-6 and IL-15 enhances anti-tumor activity through augmented NK cytotoxicity. Cancer Lett 272: 285-295, 2008.

17. Huyan T, Li Q, Yang H, Jin ML, Zhang MJ, Ye LJ, Li J, Huang QS and Yin DC: Protective effect of polysaccharides on simulated microgravity-induced functional inhibition of human NK cells. Carbohydr Polym 101: 819-827, 2014.

18. Cheng KC, Huang HC, Chen JH, Hsu JW, Cheng HC, Ou CH, Yang WB, Chen ST, Wong CH and Juan HF: Ganoderma lucidum polysaccharides in human monocytic leukemia cells: From gene expression to network construction. BMC Genomics 8: 411-428, 2007.

19. Ng ML and Yap AT: Inhibition of human colon carcinoma development by lentinan from shiitake mushrooms (Lentinus edodes). J Altern Complement Med 8: 581-589, 2002.

20. Ren M, Ye L, Hao X, Ren Z, Ren S, Xu K and Li J: Polysaccharides from Tricholoma matsutake and Lentinus edodes enhance 5-fluorouracil-mediated $\mathrm{H} 22$ cell growth inhibition. J Tradit Chin Med 34: 309-316, 2014. 